\title{
Phosphoramide Mustard
}

National Cancer Institute

\section{Source}

National Cancer Institute. Phosphoramide Mustard. NCI Thesaurus. Code C29322.

One of a number of chemically-related alkylating agents with antineoplastic properties.

The prototype of this group of agents is cyclophosphamide. Most phosphoramide

mustards are administered as prodrugs that undergo reductive activation in hypoxic

environments to yield cytotoxic metabolites. These agents alkylate and crosslink DNA,

resulting in inhibition of DNA replication. Phosphoramide mustards are also

immunosuppressants, mutagens and teratogens. ( $\mathrm{NCl04)}$ 